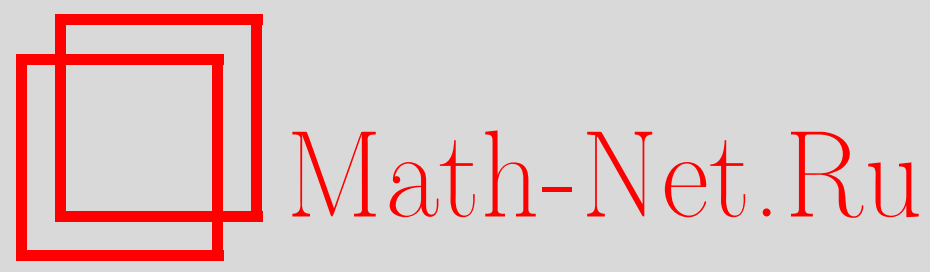

В. М. Тюрин, К обратимости линейных дифференциальных операторов с замкнутой областью значений и пуассоновскими коэффициентами, Матем. заметки, 1999, том 65, выпуск 1, 143-147

DOI: https://doi.org/10.4213/mzm1035

Использование Общероссийского математического портала Math-Net.Ru подразумевает, что вы прочитали и согласны с пользовательским соглашением http://www.mathnet.ru/rus/agreement

Параметры загрузки:

IP : 18.207.199.55

26 апреля 2023 г., $16: 37: 40$

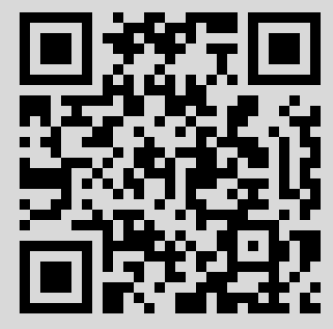




\section{К ОБРАТИМОСТИ ЛИНЕЙНЫХ ДИФФЕРЕНЦИАЛЬНЫХ ОПЕРАТОРОВ С ЗАМКНУТОЙ ОБЛАСТЬЮ ЗНАЧЕНИЙ И ПУАССОНОВСКИМИ КОЭФФИЦИЕНТАМИ}

\section{В. М. Тюрин}

Изучаются свойства обратимости и инъективности линейных дифференциальных операторов с замкнутой областью значений и пуассоновскими коэффициентами с позиции их эквивалентности в некоторых пространствах векторфункций на оси.

Библиограффия: 6 названий.

Пусть $X$ - банахово пространство; $C=C(\mathbb{R}, X)$ - нормированное пространство непрерьвных ограничений функций $u: \mathbb{R} \rightarrow X$ c sup-нормой; $M^{p}=M^{p}(\mathbb{R}, X)$ - нормированное пространство сильно измеримых функций $u: \mathbb{R} \rightarrow X$ с нормой

$$
\|u\|_{M^{p}}=\sup _{t \in \mathbb{R}}\left(\int_{t}^{t+1}\|u(s)\|^{p} d s\right)^{1 / p}<\infty, \quad p \geqslant 1 ;
$$

$L^{p}=L^{p}(\mathbb{R}, X)$ - пространство Лебега сильно измеримых функций $u: \mathbb{R} \rightarrow X$ с обычной нормой $(1 \leqslant p \leqslant \infty) ; A(t) \in C(\mathbb{R}, \operatorname{Hom}(X, X))$, где $\operatorname{Hom}(X, X)$ - пространство линейных ограниченных операторов, действующих в $X$, с равномерной топологией; $F=F(\mathbb{R}, X)$ - одно из пространств $C, M^{p}, L^{p} ; F_{*}=F\left(\mathbb{R}, X^{*}\right)\left(X^{*}\right.$ - сопряженное пространство к $X)$.

Рассмотрим выражение $\mathscr{L}=d / d t-A(t)$. Ему отвечает замкнутый неограниченный дифференциальный оператор $\mathscr{L}: D(\mathscr{L}, F) \rightarrow F$, если область определения задается равенством

$$
D(\mathscr{L}, F)=\{u \mid u \in F, \mathscr{L} u \in F\} .
$$

Следуя [1], оператор $\mathscr{L}: D(\mathscr{L}, F) \rightarrow F$ будем называть корректныц, если существует постоянная $k>0$ такая, что для любого $u \in D(\mathscr{L}, F)$ справедлива оценка

$$
\|u\|_{F} \leqslant k\|\mathscr{L} u\|_{F}
$$

Если оператор $\mathscr{L}: D(\mathscr{L}, F) \rightarrow F$ обратим, т.е. уравнение $\mathscr{L} u=f$ однозначно разрешимо в $F$ при любом $f \in F$, то по теореме Банаха обратньй оператор $\mathscr{L}^{-1}: F \rightarrow D(\mathscr{L}, F)$ непрерьвен и, следовательно, имеет место оценка (1), т.е. оператор $\mathscr{L}: D(\mathscr{L}, F) \rightarrow F$ корректен. Обратное утверждение, вообще говоря, неверно. Из корректности оператоpa $\mathscr{L}: D(\mathscr{L}, F) \rightarrow F$, вообще говоря, не следует его обратимость (см. [1], [2, с. 174, 176]). 
В конечномерном пространстве $X$ для оператора $\mathscr{L}: D(\mathscr{L}, C) \rightarrow C$ с устойчивым по Пуассону коэффициентом $A(t)$ обратимость эквивалентна его корректности [1], [2, с. 176]. В данной заметке изучается обратимость дифференциальных операторов такого вида, имеюших замкнутую область значений $\operatorname{Im} \mathscr{L}$. Отметим, что конкретные условия обратимости линейных дифференциальных операторов $\mathscr{L}: D\left(\mathscr{L}, L^{p}\right) \rightarrow L^{p}$ приведены в [3].

Оператор $\mathscr{L}: D(\mathscr{L}, F) \rightarrow F$ назовем $\Phi_{0}$-оператором, если область значений $\operatorname{Im} \mathscr{L}$ и линейное многообразие

$$
X_{0 F}=\{u(0) \mid \mathscr{L} u=0, u \in F\} \subseteq X
$$

замкнуты.

Отметим, что многообразие $X_{0 F}$ может быть незамкнутьм $[4$, с. 118$]$. В дальнейшем всюду считаем $A(t)$ устойчивой по Пуассону функцией.

Tеорема 1. Оператор $\mathscr{L}: D(\mathscr{L}, F) \rightarrow F$ является $\Phi_{0}$-оператором тогда и только тогда, когда он корректен.

ДоказАтельство. Пусть $\mathscr{L}: D(\mathscr{L}, F) \rightarrow F$ есть $\Phi_{0}$-оператор. Так как область значений $\operatorname{Im} \mathscr{L}$ замкнута и многообразие $X_{0 F}$ также замкнуто, из теоремы Банаха об открытом отображении вытекает существование постоянной $\gamma>0$ такой, что для любой функции $u \in D(\mathscr{L}, F)$ с условием $u(-\xi)=0(\xi \in \mathbb{R})$ имеет место неравенство

$$
\|u\|_{F} \leqslant \gamma\left\|\mathscr{L}_{\xi} u\right\|_{F}, \quad \mathscr{L}_{\xi}=\frac{d}{d t}-A(t+\xi)
$$

(подробнее см. [4, с. 117, 147]).

Возьмем $u \in D(\mathscr{L}, F),-\xi \neq \omega \in \mathbb{R}$ и положим

$$
v(t)=(u(t+\xi)-u(t+\xi-\omega)) \varphi(t, \omega, T) \sin \frac{\pi(t+\xi)}{2(\omega+\xi)},
$$

где $\varphi: \mathbb{R} \rightarrow[0,1]$ есть гладкое отображение с носителем $\operatorname{supp} \varphi \subseteq[\omega-2 T, \omega+2 T]$, причем $\varphi(t, \omega, T)=1$ при $|t-\omega| \leqslant T$, а производная по $t$ удовлетворяет неравенству $|\dot{\varphi}| \leqslant 2 / T$ $(T>0)$. Очевидно, $v \in D(\mathscr{L}, F)$ и $v(-\xi)=0$. Поэтому для функции $v$ справедлива оценка (2), из которой при $F=L^{p}(p \geqslant 1)$ имеем

$$
\begin{aligned}
\|v\|_{L^{p}} \leqslant & \gamma\left(\int_{-2 T}^{2 T}\|f(t+\xi+\omega)-f(t+\xi)\|^{p} d t\right)^{1 / p}+\frac{4 \gamma}{T}\|u\|_{L^{p}}+\frac{\pi \gamma}{|\omega+\xi|}\|u\|_{L^{p}} \\
& +\gamma \sup _{|t| \leqslant 2 T}\|A(t+\xi+\omega)-A(t+\xi)\| \cdot\|u\|_{L^{p}}, \quad f=\mathscr{L} u .
\end{aligned}
$$

Далее воспользуемся известньм неравенством для решения линейного дифференциального уравнения

$$
\|u(t)\| \leqslant\left(l^{-1} \int_{I}\|u(t)\| d t+\int_{I}\|f(t)\| d t\right) \exp \left(\int_{I}\|A(t)\| d t\right),
$$


где $I$ - интервал длины $l$, содержащий точку $t \in \mathbb{R}$. Применение оценок $(3),(4)$ к функции $v$ приводит к формуле

$$
\begin{aligned}
\|u(\xi+\omega)-u(\xi)\| \leqslant & \left(\frac{s_{1}}{T}+\frac{s_{2}}{|\omega+\xi|}\right)\|u\|_{L^{p}} \\
& +s_{3}\left(\int_{-2 T}^{2 T}\|f(t+\xi+\omega)-f(t+\xi)\|^{p} d t\right)^{1 / p} \\
& +s_{3} \sup _{|t| \leqslant 2 T}\|A(t+\xi+\omega)-A(t+\omega)\| \cdot\|u\|_{L^{p}} \\
s_{1}=4 \gamma+4 & \exp \left(\|A\|_{C}\right), \quad s_{2}=\frac{\pi}{4} s_{1}, \quad s_{3}=\frac{s_{1}}{4} .
\end{aligned}
$$

Положив в (5) $f=0, \xi=0$, имеем

$$
\|u(\omega)-u(0)\| \leqslant\left(\frac{s_{1}}{T}+\frac{s_{2}}{|\omega|}\right)\|u\|_{L^{p}}+s_{3} \sup _{|t| \leqslant 2 T}\|A(t+\omega)-A(t)\| \cdot\|u\|_{L^{p}} .
$$

Предположим, что $X_{0 L^{p}} \neq 0$, т.е. однородное уравнение $\mathscr{L} u=0$ имеет ненулевое решение $u \in L^{p}$. Поскольку $A(t)$ - устойчивая по Пуассону операторная функция, сушествует последовательность $\omega_{j} \in \mathbb{R}$ такая, что $\omega_{j} \rightarrow \infty$ и $A\left(t+\omega_{j}\right)$ локально сходится к $A(t)$ в $C(\mathbb{R}, \operatorname{Hom}(X, X))$ при $j \rightarrow \infty$. Так как функция $v_{j}=u(t) \sin \left(\pi t /\left(2 \omega_{j}\right)\right)$ принадлежит $D\left(\mathscr{L}, L^{p}\right)$ и $v_{j}(0)=0$, согласно $(2)$

$$
\left\|v_{j}\right\|_{L^{p}} \leqslant \frac{\pi \gamma}{2 \omega_{j}}\|u\|_{L^{p}}
$$

Отсюда по формуле (4) имеем

$$
\left\|v_{j}\left(\omega_{j}\right)\right\| \leqslant\left(\left\|v_{j}\right\|_{L^{p}}+\frac{\pi}{2 \omega_{j}}\|u\|_{L^{p}}\right) \exp \left(\|A\|_{C}\right) \leqslant\left(\frac{\pi \gamma}{2 \omega_{j}}+\frac{\pi}{2 \omega_{j}}\right)\|u\|_{L^{p}} \exp \left(\|A\|_{C}\right) .
$$

В силу замкнутости $X_{0 L^{p}}$ с некоторой постоянной $s>0$ на основе $(6),(7)$ получаем неравенства

$$
\begin{aligned}
s^{-1}\|u\|_{L^{p}} \leqslant\|u(0)\| \leqslant & \left(\frac{s_{1}}{T}+\frac{s_{2}}{\omega_{j}}\right)\|u\|_{L^{p}}+s_{3} \sup _{|t| \leqslant 2 T}\left\|A\left(t+\omega_{j}\right)-A(t)\right\| \cdot\|u\|_{L^{p}} \\
& +\left(\frac{\pi \gamma}{2 \omega_{j}}+\frac{\pi}{2 \omega_{j}}\right)\|u\|_{L^{p}} \exp \left(\|A\|_{C}\right) .
\end{aligned}
$$

Предельньй переход по $j \rightarrow \infty$ в (8) дает $T \leqslant s s_{1}$ при любом $T>0$, а это невозможно. Следовательно, $X_{0 L^{p}}=0$ и $\operatorname{ker}\left(\mathscr{L}: D\left(\mathscr{L}, L^{p}\right) \rightarrow L^{p}\right)$. Так как область значений оператора $\mathscr{L}: D\left(\mathscr{L}, L^{p}\right) \rightarrow L^{p}$ замкнута, по теореме Банаха он корректен.

Случаи $F=M^{p}(p \geqslant 1), F=L^{\infty}, F=C$ рассматриваются аналогично.

Если оператор $\mathscr{L}: D(\mathscr{L}, F) \rightarrow F$ корректен, то его область значений, очевидно, замкнута, а многообразие $X_{0 F}=0$, т.е. $\mathscr{L}: D(\mathscr{L}, F) \rightarrow F$ является $\Phi_{0}$-оператором. Теорема доказана. 
СлЕДСТВИЕ 1. Сюрвективный оператор $\mathscr{L}: D(\mathscr{L}, F) \rightarrow F$ обратим, если и только если множество $X_{0 F}$ замкнуто в $X$.

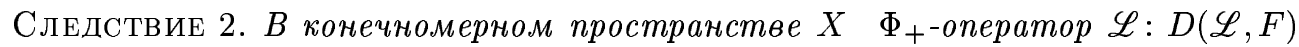
$\rightarrow$ F обратим.

Кроме теоремы 1 при доказательстве этого следствия нужно воспользоваться результатом работы [5] об эквивалентности свойств корректности для операторов $\mathscr{L}$ : $D(\mathscr{L}, F) \rightarrow F\left(F \subset\left\{C, L^{\infty}, M^{p}, L^{p}\right\}, p \geqslant 1\right)$.

СлЕДСТВИЕ 3. Приводимые ниже условия равносильны:

1) onepamop $\mathscr{L}: D(\mathscr{L}, F) \rightarrow F$ ecmb $\Phi_{0}$-onepamop;

2) оператор $\mathscr{L}: D(\mathscr{L}, F) \rightarrow F$ корректен;

3) оператор $\mathscr{L}: D(\mathscr{L}, F) \rightarrow F$ является $\Phi_{+}$-оператором.

СлеДСТвИЕ 4. Если один из операторов $\mathscr{L}: D(\mathscr{L}, C) \rightarrow C, \mathscr{L}: D\left(\mathscr{L}, L^{\infty}\right) \rightarrow L^{\infty}$, $\mathscr{L}: D\left(\mathscr{L}, M^{p}\right) \rightarrow M^{p}, \mathscr{L}: D\left(\mathscr{L}, L^{p}\right) \rightarrow L^{p}(p \geqslant 1)$ есть $\Phi_{+}$-оператор, то остальные

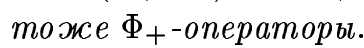

ДокАЗАТЕЛьство. Пусть, например, $\mathscr{L}: D\left(\mathscr{L}, L^{p}\right) \rightarrow L^{p}$ является $\Phi_{+}$-оператором. Согласно следствию 3 этот оператор корректен. Но тогда остальные дифференциальные операторы также корректны [5] (см. доказательство следствия 2), а следовательно, есть $\Phi_{+}$-операторы.

Теорема 2. В рефлексивном пространстве $X$ дифференииальные $\mathscr{L}: D(\mathscr{L}, C) \rightarrow C, \mathscr{L}: D\left(\mathscr{L}, L^{\infty}\right) \rightarrow L^{\infty}, \mathscr{L}: D\left(\mathscr{L}, M^{p}\right) \rightarrow M^{p}, \mathscr{L}: D\left(\mathscr{L}, L^{p}\right) \rightarrow L^{p}$ обратимы.

ДокАЗАТЕЛЬСТво приведем с некоторыми сокращениями. Предположим, что опеpaтор $\mathscr{L}: D(\mathscr{L}, C) \rightarrow C$ есть $\Phi$-оператор. По следствию 3 этот оператор корректен. Вместе с ним корректен оператор $\mathscr{L}: D\left(\mathscr{L}, L^{2}\right) \rightarrow L^{2}$. Двойственный оператор $\mathscr{L}^{*}:$ $D\left(\mathscr{L}^{*}, L_{*}^{2}\right) \rightarrow L_{*}^{2}$ служит сопряженньм оператором к оператору $\mathscr{L}: D\left(\mathscr{L}, L^{2}\right) \rightarrow L^{2}$ относительно пространства $L_{*}^{2}=L^{2}\left(\mathbb{R}, X^{*}\right)$. По теореме Банаха область значений $\operatorname{Im}\left(\mathscr{L}^{*}: D\left(\mathscr{L}^{*}, L_{*}^{2}\right) \rightarrow L_{*}^{2}\right)$ совпадает с $L_{*}^{2}$ (напомним, что оператор $\mathscr{L}: D\left(\mathscr{L}, L^{2}\right) \rightarrow L^{2}$ замкнут, а $D\left(\mathscr{L}, L^{2}\right)$ плотно в $\left.L^{2}\right)$. Ядро $\operatorname{ker}\left(\mathscr{L}^{*}: D\left(\mathscr{L}^{*}, L_{*}^{2}\right) \rightarrow L_{*}^{2}\right)$ конечномерно, ибо оно входит в ядро оператора $\mathscr{L}^{*}: D\left(\mathscr{L}^{*}, C^{*}\right) \rightarrow C^{*}$. Поэтому многообразие $X_{0 L_{*}^{2}}$ замкнуто. Согласно теореме 1 оператор $\mathscr{L}^{*}: D\left(\mathscr{L}^{*}, L_{*}^{2}\right) \rightarrow L_{*}^{2}$ корректен. Но тогда будет корректен и оператор $\mathscr{L}^{*}: D\left(\mathscr{L}^{*}, C_{*}\right) \rightarrow C_{*}$, что вместе с корректностью оператора $\mathscr{L}: D(\mathscr{L}, C) \rightarrow C$ влечет их обратимость [1], [6].

Пусть оператор $\mathscr{L}: D\left(\mathscr{L}, L^{p}\right) \rightarrow L^{p}$ является $\Phi$-оператором. В силу следствия 3 он корректен. Рассмотрим в $X^{*}$ оператор $\mathscr{L}^{*}=d / d t+A^{*}(t)$. Двойственным к нему будет оператор $\mathscr{L}=d / d t-A(t)$. Далее, из корректности оператора $\mathscr{L}: D\left(\mathscr{L}, L^{p}\right) \rightarrow L^{p}$ следует корректность оператора $\mathscr{L}: D(\mathscr{L}, C) \rightarrow C$. В этом случае оператор $\mathscr{L}^{*}: D\left(\mathscr{L}^{*}, L_{*}^{p}\right)$ $\rightarrow L_{*}^{p}$ сюръективен. Так как ядро $\operatorname{ker}\left(\mathscr{L}^{*}: D\left(\mathscr{L}^{*}, L_{*}^{p}\right) \rightarrow L_{*}^{p}\right)$ конечномерно, для оператора $\mathscr{L}^{*}: D\left(\mathscr{L}^{*}, L_{*}^{p}\right) \rightarrow L_{*}^{p}$ выполнены условия теоремы 1 . Следовательно, этот оператор корректен.

Отсюда выводим, что оператор $\mathscr{L}^{*}: D\left(\mathscr{L}^{*}, C_{*}\right) \rightarrow C_{*}$ также корректен. Как отмечалось вьше, в такой ситуации оператор $\mathscr{L}: D(\mathscr{L}, C) \rightarrow C$ обратим, что эквивалентно обратимости оператора $\mathscr{L}: D\left(\mathscr{L}, L^{p}\right) \rightarrow L^{p}$. 
Если $F=M^{p}$, то доказательство проведем следующим образом. Согласно следствиям 3,4 оператор $\mathscr{L}: D(\mathscr{L}, C) \rightarrow C$ корректен. Так как

$$
\operatorname{ker}\left(\mathscr{L}^{*}: D\left(\mathscr{L}^{*}, C_{*}\right) \rightarrow C_{*}\right) \subseteq \operatorname{ker}\left(\mathscr{L}^{*}: D\left(\mathscr{L}^{*}, M_{*}^{p}\right) \rightarrow M_{*}^{p}\right),
$$

оператор $\mathscr{L}: D(\mathscr{L}, C) \rightarrow C$ есть $\Phi$-оператор. По доказанному такой дифференциальньй оператор обратим. Вместе с ним будет обратим и оператор $\mathscr{L}: D\left(\mathscr{L}, M^{p}\right) \rightarrow M^{p}$.

Аналогично доказьвается теорема для оператора $\mathscr{L}: D\left(\mathscr{L}, L^{\infty}\right) \rightarrow L^{\infty}$. Теорема доказана.

ЗАмЕчАниЕ. Сходные результаты справедливы для разностного оператора вида

$$
R x(n)=x(n+1)-B(n) x(n), \quad n \in \mathbb{Z} .
$$

\section{СПИСОК ЦИТИРОВАННОЙ ЛИТЕРАТУРЫ}

[1] ЖКиков В. В. Некоторые вопросы допустимости и дихотомии. Принцип усреднения // Изв. АН СССР. Сер. матем. 1976. Т. 40. №6. С. 1380-1408.

[2] Левитан Б. М., Жииков В. В. Почти периодические функции и дифференциальные уравнения. М.: Изд-во МГУ, 1978.

[3] Баскаков А. Г. Некоторые условия обратимости линейных дифференциальньх и разностных операторов // Докл. РАН. 1993. Т. 333. № 3. С. 282-284.

[4] Maccepa X., Шеффер X. Линейные дифференциальные уравнения и функциональные пространства. М.: Мир, 1970.

[5] Тюрин В. М. Об обратимости оператора $d / d t-A(t)$ в некоторых функциональных пространствах // Матем. заметки. 1979. Т. 25. № 4. С. 585-590.

[6] Тюрин В. М. Об обратимости линейных дифференциальных операторов в некоторых функциональных пространствах // Сиб. матем. ж. 1991. Т. 32. №1. С. 160-165.

Липецкий государственный технический университет

Поступило 28.10 .96

Исправленный вариант

20.04 .98 